\title{
Quantitative Analysis and In Silico Molecular Docking Screening for Acetylcholinesterase Inhibitor and ADME Prediction of Coumarins and Carbazole Alkaloids from Clausena harmandiana
}

\author{
Bunleu Sungthong ${ }^{1}$, Kingkaeo Sithon ${ }^{(2}$, Panyada Panyatip $\oplus^{3}$, \\ Sarin Tadtong $\oplus^{4}$, Nadtanet Nunthaboot $\oplus^{5}$ and Ploenthip Puthongking $\oplus^{6,7^{*}}$
}

\author{
${ }^{1}$ Pharmaceutical Chemistry and Natural Products Research Unit, Faculty of Pharmacy, Mahasarakham \\ University, Mahasarakham 44150, Thailand \\ ${ }^{2}$ Pharmaceutical Chemistry and Natural Products, Graduate school, Khon Kaen University, \\ Khon Kaen 40002, Thailand \\ ${ }^{3}$ Melatonin Research Group, Faculty of Pharmaceutical Sciences, Khon Kaen University, Khon Kaen \\ 40002, Thailand \\ ${ }^{4}$ Faculty of Pharmacy, Srinakharinwirot University, Nakhon-nayok 26120, Thailand \\ ${ }^{5}$ Center of Excellence for Innovation in Chemistry (PERCH-CIC), Faculty of Science, Mahasarakham \\ University, Maha Sarakham 44150, Thailand \\ ${ }^{6}$ Center for Research and Development of Herbal Health Products, Faculty of Pharmaceutical Sciences, \\ Khon Kaen University, Khon Kaen 40002, Thailand \\ ${ }^{7}$ Department of Pharmaceutical Chemistry, Faculty of Pharmaceutical Sciences, Khon Kaen University, \\ Khon Kaen 40002, Thailand
}

(Received July 01, 2021; Revised September 19, 2021; Accepted September 21, 2021)

\begin{abstract}
Coumarins and carbazole alkaloids are natural compounds, often with neurological properties, especially as acetylcholinesterase inhibitors. In order to screen the candidate anti-acetylcholinesterase agents, in-silico molecular docking was performed aiming to predict the binding mechanism and the results were used to identify pharmacophores of coumarins and carbazole alkaloids from Clausena harmandiana as AChE inhibitory activity. All isolated compounds are xanthoxyletin (1), dentatin (2), nordentatin (3), clausarin (4), 7-methoxymukonal (5), 7-methoxyheptaphylline (6) and heptaphylline (7) displayed the stable binding interactions with Torpedo californica acetylcholinesterase (TcAChE) enzyme via $\pi-\pi$ interactions, hydrogen bonds and hydrophobic interactions. All compounds exhibited the interaction with the peripheral anionic site of $T c \mathrm{AChE}$ same manner of standard anti-alzheimer drugs. ADME prediction revealed that all compounds met the requirement of drug likeness of Lipinski's rule of five and had high CNS absorption, excepted $\mathbf{4}$ should be structure modification to improve CNS penetration. Besides $\mathbf{4 , 6}$ and $\mathbf{7}$ displayed the highest acetylcholinesterase inhibitor activity among the other compounds. Interestingly, $\mathbf{6}$ had a high yield $(58.01 \mathrm{mg} / \mathrm{g}$ extract) from $C$. harmandiana. It should be a candidate as an anti-acetylcholinesterase inhibitor agent.
\end{abstract}

Keywords: Clausena harmandiana; molecular docking; coumarins; carbazole alkaloids; Alzheimer's disease; ADME (c) 2021 ACG Publications. All rights reserved.

\footnotetext{
*Corresponding author: E-Mail: pploenthip@kku.ac.th; Phone: +66-43-203149
} 


\section{Introduction}

Alzheimer's disease (AD) is the most common form of dementia; the symptoms of which include memory loss, difficulty solving problems, disorientation in time and space, among others [1]. Although the etiology of $\mathrm{AD}$ is not completely known, several diverse hallmarks such as $\beta$-amyloid $(\mathrm{A} \beta)$ deposits, tau protein aggregation, oxidative stress, or low levels of acetylcholine (ACh) play significant roles in the pathophysiology of the disease. The cholinergic hypothesis was the first theory proposed to explain AD and has since led to the development of the only drugs currently approved to treat mild to moderate AD [2]. This theory was based on the loss of cholinergic activity that is commonly observed in the brains of AD patients. Acetylcholinesterase inhibitors (AChEIs) initially showed promise in reversing the memory impairment in $\mathrm{AD}$ patients, drug substances are currently used such as donepezil, rivastigmine, galantamine, and tacrine [3].

The crystal structure AChE is known that the catalytic anionic site (CAS) is the major binding site targeted for AD treatment and amino acids in the active site are the main part of this studies. Trp84 is an amino acid of CAS clusters that has been reported to interact with AChE $[4,5]$. It also interacts with another important cluster, the peripheral anionic site (PAS) that is concerned with $\beta$-amyloid aggregation [6]. Therefore, molecular docking is the primary screening method to find the potential AChE inhibition of compounds.

Several compounds from natural products are interesting as anti-Alzheimer agents. Especially, compounds isolated from the plant Clausena spp. have been reported to have neuroprotective activities. For instance, the various parts of Clausena lansium, including compounds found in the fruits, showed significant neuroprotective activity with $\mathrm{EC}_{50}$ values ranging from 0.36 to $10.69 \mu \mathrm{M}$ [7], and isolated compounds from stems showed a neuroprotective effect on a neuroprotective effect on Pheochromocytoma (PC12) cells at the concentration of $10 \mu \mathrm{M}[8,9]$. The major chemical constituents from Clausena spp. were classified as coumarins and carbazole alkaloids [10]. Nordentatin, the major coumarin constituent, displayed significantly more branching numbers of the neurites than in the control $(p<0.05)$ and showed no neurotoxicity on cultured P19 neurons $\left(\mathrm{IC}_{50}>10 \mu \mathrm{M}\right)$ [11]. Additionally, coumarins from Angelica gigas, including 7demethylsuberosine, umbelliferone, isoimperatorin, xanthyletin, 7-methoxy-5-prenyloxycoumarin, decursin, 7-hydroxy-6-(2-(R)-hydroxy-3-methylbut-3-enyl-coumarin, nodakenin, and peucedanone showed potent AChE inhibition [12]. In addition, carbazole alkaloids including euchrestifoline [13] and mahanimbine [14] showed AChE inhibitory activity with $\mathrm{IC}_{50} 93.1 \mu \mathrm{M}$ and $0.03 \mathrm{mg} / \mathrm{mL}$, respectively. Besides AChE inhibitory activity, they possess several biological activities such as antibacterial, anti-inflammatory [15], anticoagulant [16], anticancer [17, 18], and antimicrobial activities [19]. In Thailand, Clausena harmandiana is widely distributed in the northeast and is used for the folk medicine for treatment of fever and antipyretic. Our group succeed to isolate seven compounds from the root bark of $C$. harmandiana, consisting of four coumarins are xanthoxyletin (1), dentatin (2), nordentatin (3), clausarin (4), and three carbazole alkaloids are 7methoxymukonal (5), 7-methoxyheptaphylline (6) and heptaphylline (7) (Table 1) [18]. In order to screen candidate anti-Alzheimer's agents from natural products, in silico investigation of molecular docking was carried out to explore the feasibility of using the major natural components from $C$. harmandiana to interact the AChE enzyme. The quantitative analysis of coumarins and carbazole alkaloids contains in $C$. harmandiana were also analysed.

\section{Materials and Methods}

\subsection{Plant Material}

Roots of $C$. harmandiana were harvested from Khon Kaen province, Thailand in February 2018 and a voucher specimen (No KKU 21145) was deposited at the Faculty of Pharmaceutical Sciences, Khon Kaen University. Air-dried root barks of C. harmandiana $(5.2 \mathrm{~kg})$ were pulverized and macerated with $95 \%$ ethanol at room temperature overnight. The ethanolic extract was then filtered and evaporated to give crude extract ( $370.54 \mathrm{~g})$. The crude extract $(31.57 \mathrm{~g})$ was further purified by open column chromatography technique affording four coumarins (1-4) and three carbazole alkaloids (5-7). Structure elucidation of all compounds were accomplished on ${ }^{1} \mathrm{H}-\mathrm{NMR}$ and ${ }^{13} \mathrm{C}-\mathrm{NMR}$. All spectra corresponded in all aspects to our previous results [18]. 
AChE inhibitory effects of coumarins and carbazole alkaloids from C. harmandiana

\subsection{Chemical Constituent Analysis}

The crude extract was reconstituted with $50 \%$ ethanol at a concentration of $1 \mathrm{mg} / \mathrm{mL}$ and dissolved by ultrasonication for $15 \mathrm{~min}$ and then filtered through $0.45 \mu \mathrm{m}$ PTFE (ANPEL Laboratory Technologies, Shanghai, PR China).

Table 1. Structure of the isolated coumarins and carbazole alkaloid from C. harmandiana<smiles>[R1]c1cc2c(Br)c3c(c([Z8])c2oc1=O)OC(C)(C)C=C3</smiles>

\begin{tabular}{|c|c|c|c|c|c|}
\hline Compounds & Name & $\mathbf{R}_{\mathbf{1}}$ & $\mathbf{R}_{2}$ & $\mathbf{R}_{\mathbf{3}}$ & $\begin{array}{c}\text { Amount } \\
\text { (mg/g extract) }\end{array}$ \\
\hline 1 & Xanthoxyletin & $\mathrm{H}$ & $\mathrm{H}$ & $\mathrm{OCH}_{3}$ & 27.60 \\
\hline 2 & Dentatin & $\mathrm{C}\left(\mathrm{CH}_{3}\right)_{2} \mathrm{CH}=\mathrm{CH}_{2}$ & $\mathrm{H}$ & $\mathrm{OCH}_{3}$ & 13.63 \\
\hline 3 & Nordentatin & $\mathrm{C}\left(\mathrm{CH}_{3}\right)_{2} \mathrm{CH}=\mathrm{CH}_{2}$ & $\mathrm{H}$ & $\mathrm{OH}$ & 15.82 \\
\hline 4 & Clausarin & $\mathrm{C}\left(\mathrm{CH}_{3}\right)_{2} \mathrm{CH}=\mathrm{CH}_{2}$ & $\mathrm{C}\left(\mathrm{CH}_{3}\right)_{2} \mathrm{CH}=\mathrm{CH}_{2}$ & $\mathrm{OH}$ & 3.73 \\
\hline Compounds & Name & $\mathbf{R}_{1}$ & $\mathbf{R}_{2}$ & $\mathbf{R}_{3}$ & $\begin{array}{c}\text { Amount } \\
\text { (mg/g extract) }\end{array}$ \\
\hline 5 & 7-Methoxy mukonal & $\mathrm{H}$ & $\mathrm{OCH}_{3}$ & $\mathrm{H}$ & 21.72 \\
\hline 6 & $\begin{array}{l}\text { 7-Methoxy } \\
\text { heptaphylline }\end{array}$ & $\mathrm{CH}_{2} \mathrm{CH}=\mathrm{C}\left(\mathrm{CH}_{3}\right)_{2}$ & $\mathrm{OCH}_{3}$ & $\mathrm{H}$ & 58.01 \\
\hline 7 & Heptaphylline & $\mathrm{CH}_{2} \mathrm{CH}=\mathrm{C}\left(\mathrm{CH}_{3}\right)_{2}$ & $\mathrm{H}$ & $\mathrm{H}$ & 36.76 \\
\hline
\end{tabular}

All standard compounds (1-7) were prepared in the same manner as the crude extract at a concentration of 2$1,000 \mu \mathrm{g} / \mathrm{mL}$. The analytical method of was performed on a Primaide 1000 series (Hitachi, Tokyo, Japan) coupled with photodiode array detector. The analysis was carried out using ZORBAX Eclipse XDB HPLC Columns $(250 \times 4.6 \mathrm{~mm}, 5 \mu \mathrm{m})$ with an Eclipse XDB HPLC guard column $(13 \times 4.6 \mathrm{~mm}, 5 \mu \mathrm{m})$ (Agilent Technology, CA, USA) with a flow rate of $1 \mathrm{~mL} / \mathrm{min}$. The gradient of the mobile phase of analytical method was slightly modified from previous method [10]. It consisted of $0.05 \%$ trifluoro-acetic acid (A) (SigmaAldrich, St. Louise, MO, USA) and acetonitrile (B) (RCI Labscan, Bangkok, Thailand): 0-10 min, 40-50 $\% \mathrm{~B}$; 10-23 min, 50-60 \% B; 23-35 min, 60-100 \%B; 35-37 min, $100 \% \mathrm{~B}$; 37-40 min, 100-40\% \%; 40-45 min, $40 \% \mathrm{~B}$; then held for $10 \mathrm{~min}$ prior to next injection. The analytical method was validated according to ICH guideline Q2(R1) [20]. Limit of detection (LOD) and limit of quantitation (LOQ) were calculated based on the standard deviation of the response and the slope $(\mathrm{LOD}=3.3 \times \mathrm{SD} / \mathrm{S}$ and $\mathrm{LOQ}=10 \times \mathrm{SD} / \mathrm{S}$; where $\mathrm{SD}=$ standard deviation of response and $\mathrm{S}$ is slope of the calibration curve). A 95\% confidence interval at was also 
evaluated. Percentage recovery was calculated and the amount found compared with the amount added into the samples. Stability of standard solution at ambient temperature (approximately $25^{\circ} \mathrm{C}$ ) was also evaluated at 48 hours after preparation.

\subsection{In Vitro Inhibition Study on Acetylcholinesterase (AChE) Enzyme}

The inhibitory effect on AChE was modified from Ellman's spectrophotometric method [21]. Briefly, $25 \mu \mathrm{L}$ of 0.2 units/mL AChE from Electrophorus electricus in $50 \mathrm{mM}$ TRIS-HCl buffer $\mathrm{pH} 8.0$ and $25 \mu \mathrm{L}$ of sample were mixed in 96-well plate. All samples were dissolved with methanol to $10 \mathrm{mg} / \mathrm{ml}$ stock solution and diluted with the buffer to tested concentration. Then, $25 \mu \mathrm{L}$ of $1.5 \mathrm{mM}$ acetylthiocholine iodide (ATCI) and $125 \mu \mathrm{L}$ of $1.0 \mathrm{mM}$ 5,5'-dithiobis-(2-nitrobenzoic acid) (DTNB) were added. The absorbance was measured at $405 \mathrm{~nm}$ by a microplate reader (EnSight Multimode Microplate Reader, PerkinElmer Inc., Waltham, MA, USA). All isolated compounds were evaluated their median inhibitory concentration ( $\left.\mathrm{IC}_{50}\right)$ and galantamine was used as a standard inhibitor.

\subsection{Docking Validation}

The crystal structure of Torpedo californica acetylcholinesterase (TcAChE) complexed with donepezil inhibitor [22] (PDB code: 1EVE) was downloaded from the RCSB Protein Databank. Water molecules were preliminary removed, and hydrogen atoms were then added to the enzyme by Discovery Studio 5.0 (Accelrys, San Diego, USA). The donepezil molecule was minimized using HyperChem7.0 software (Hypecube, Gainesville, FL, USA) by the Semi-empirical, Polak-Ribiere conjugate gradient method (RMSD $<0.1 \mathrm{kcal} / \mathrm{A} m o l)$. Docking calculations were carried out using AutoDock4.2 [23]. A grid box was generated by AutoDockTools [24] with the dimensions $60 \AA \times 60 \AA \times 60 \AA$ with a $0.375 \AA$ grid spacing. The empirical free energy function together with the Lamarckian genetic algorithm (LGA) [25] was used. The LGA protocol applied a population size of 300 individuals, while 250,000 energy evaluations were used for the 200 LGA runs. The parameters of re-docking calculations showing lowest binding energy and root-meansquare deviation (RMSD) of no more than $2.0 \AA$ were used for docking with other ligands.

\subsection{Molecular Docking Validation and Sudies}

The 3D structures of all isolated compounds were built using HyperChem7.0, and energy minimized by the semi-empirical, Polak-Ribiere conjugate gradient method (RMSD $<0.1 \mathrm{kcal} /$ Ammol). All generated 3D structures were then converted into ODB formatby Open babel [26]. Based on the parameter from the docking validation method, a grid box with dimensions of $60 \AA \times 60 \AA \times 60 \AA$ was set up by AutoGrid4.2. Docking calculations were carried out using AutoDock4.2. During the searching process, the host was treated as rigid while the guest molecule was allowed to move freely. The selected post of protein-ligand structures was visualized using PyMOL (Schrödinger LLC, New York, NY).

\subsection{ADME Properties}

Pharmacokinetic properties of all isolated compounds were analysed and screened for Absorption Distribution Metabolism and Excretion (ADME) properties by SwissADME program of the Molecular Modelling Group of the Swiss Institute of Bioinformatics [27].

\section{Results and Discussion}

\subsection{Chemical Constituents}

A simple and efficient analysis of four coumarins (xanthoxyletin (1), dentatin (2), nordentatin (3), and clausarin (4)) and three carbazole alkaloids (7-methoxymukonal (5), 7-methoxyheptaphylline (6) and heptaphylline (7)) had been previously isolated and characterized by our group [18]. 

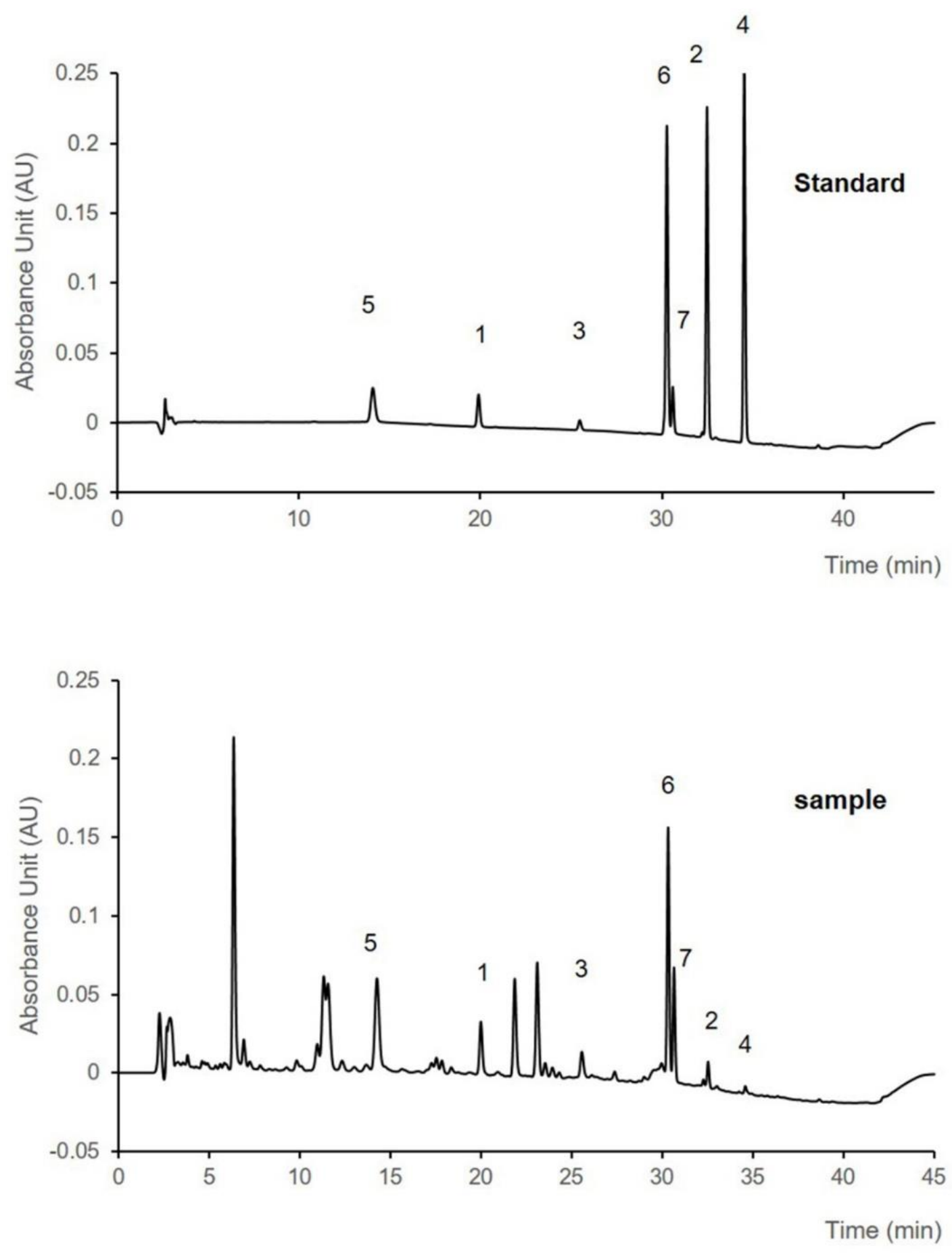

Figure 1. Chromatogram of isolated coumarins and carbazole alkaloids and crude extract of C. harmandiana

For standardization of the crude extract, the major compound in crude extract has to be determined. The fingerprint and amounts of compounds in the crude extract were used for quality control of the extract. Then, a simple and efficient analysis of the compound (1-7) was developed and validated for the 
determination in C. harmandiana crude extract. Chromatograms of a simultaneous determination of standard compounds and the crude extract are shown in Figure 1. The advantage of the proposed method is that is consisted of 2 mobile phase components comparing with 3 components. The resolution of the crucial separation of 6 and 7 was nevertheless comparable to the previous method [10]. Although they did not show the baseline separation, the validation parameters exhibited an adequate result. The amount of compound (17) ranged between $3.73-58.01 \mathrm{mg} / \mathrm{g}$ extract. 6 was the highest component found in the crude extract, while 4 was the lowest component (Table 2). The amount of each compound in the crude extract was approximately 10-30 folds higher than that used in the crude extract of the previous research [10]. We could explain that the previous study calculated on a basis of dried plant material, while the recent one calculated the quantities of each compound in the extract. The method showed a precise separation of the 7 major compounds with intraday precision in the range of $0.41-0.56 \%$ at $25 \mu \mathrm{g} / \mathrm{mL}$ (low concentration) and $0.29-0.42 \%$ at $75 \mu \mathrm{g} / \mathrm{mL}$ (high concentration). For inter-day precision, $\% \mathrm{RSD}$ of $25 \mu \mathrm{g} / \mathrm{mL}$ (low concentration) ranged from 1.39-1.83 and $\%$ RSD of $75 \mu \mathrm{g} / \mathrm{mL}$ (high concentration) ranged from 0.93-1.02. The amount and other validation parameters of analytical method of the compounds (1-7) are shown in Table 2.

\subsection{In Vitro AChE Inhibitory Activity and Molecular Intreactions Studies}

Computer-based models were evaluated for docking scores as well as key protein-ligand interactions between Torpedo californica acetylcholinesterase (TcAChE) and the isolated compounds, coumarins (1-4) and carbazole alkaloids (5-7) as shown in Table 3. The positive controls of AChE inhibitors agents were donepezil, rivastigmine, galantamine, and tacrine, which were anti-AD drugs, and these were docked into the active site of the crystal structure of $T c \mathrm{AChE}$ enzyme to validate the docking protocol. All positive controls had docking score values of $-11.30,-7.04,-9.84$ and $-7.34 \mathrm{kcal} / \mathrm{mol}$, and related to the previously reports [2830]. The interactions between all positive drugs and $T c \mathrm{AChE}$ enzyme were H-bonding interactions, hydrophobic interactions and $\pi-\pi$ interactions (Table 3). Most of ligands displayed the interaction with PAS and CAS active sites of the enzyme, meanwhile galantamine had more interaction with the catalytic triad (CT) and tacrine showed H-bonding with Phe331 only at the acyl-binding pocket site (ABP).

AChE activity of target compounds was determined by the modified from Ellman's spectrophotometric method. Galantamine was used as a reference compound. The $\mathrm{IC}_{50}$ values of the compounds are summarized in Table 3. Among coumarin and carbazole alkaloid derivatives, 4, 6 and 7 showed the highest inhibitory activity against AChE. Considering how the isolated compounds interact with the targeted AChE enzyme, the structure relationship of coumarins and carbazole alkaloids with the binding site were observed and compared from their crystallographic data in Table 3 and Figure 2. The molecular docking result showed that $\mathbf{1}$ formed two strong H-bonds between pyranone ring and Arg289 at the acyl binding pocket (AP) and another one is between methoxy group at R3 and Trp279 at PAS (Figures 2a). For 2 with the 1,1-dimethyl-2-propenyl group at R1 formed a more stable binding free energy $(\Delta \mathrm{G} ;-9.56$ $\mathrm{kcal} / \mathrm{mol}$ ) with $T c \mathrm{AChE}$ than that $\mathbf{3}$ has the same 1,1-dimethyl-2-propenyl at R1, and $\mathrm{H}$-atom at $\mathrm{R} 2$, the hydroxy of $\mathbf{3}$ or methoxy of $\mathbf{2}$ had similar value of docking score -9.20 and $-9.56 \mathrm{kcal} / \mathrm{mol}$, respectively (Figures 2b-2c). Moreover, 1,1-dimethyl-2-propenyl substituted group at R1 and R2 as found in 4, resulted in $\pi-\pi$ interaction with Tyr334 at PAS via the pyranone ring that plays an increasing the docking score as $10.43 \mathrm{kcal} / \mathrm{mol}$. The ketone group of the pyranone ring and hydroxy group at R3 of $\mathbf{4}$ also makes H-bond with Tyr121 at the PAS site and Phe330 at the CAS site (Figures 2d).

The molecular docking result for carbazole alkaloids 5-7 with $T c \mathrm{AChE}$ showed that their aromatic rings (Figures $2 \mathrm{e}-2 \mathrm{~g}$ ) had $\pi-\pi$ interactions with $T c \mathrm{AChE}$; additionally, the $\mathrm{O}$-atom from both hydroxy and formyl groups showed H-bonding interaction with PAS and ABP sites. Compound 5 established a $\pi-\pi$ interactions with Phe331 at ABP while, O-atom of the formyl group showed H-bonding with Trp279 at the PAS site and the hydroxy group with Ser286 at ABP. Compounds 6 and 7 showed $\pi-\pi$ interactions with Tyr334 at PAS, and the O-atom of the formyl group showed hydrogen bonding with Phe288 at the ABP site. Additionally, the hydroxy group exhibited hydrogen bonding with Trp279 at the PAS site. 
Table 2. Validation parameters and amount of coumarins and carbazole alkaloids of $C$. harmandiana extract

\begin{tabular}{|c|c|c|c|c|c|c|c|}
\hline Validation parameters & 1 & 2 & 3 & 4 & 5 & 6 & 7 \\
\hline \multicolumn{8}{|l|}{$\%$ RSD } \\
\hline \multicolumn{8}{|l|}{ Intra-day precision* $(\mathrm{n}=6)$} \\
\hline -low concentration $(25 \mu \mathrm{g} / \mathrm{mL})$ & 0.47 & 0.41 & 0.43 & 0.45 & 0.48 & 0.55 & 0.56 \\
\hline -high concentration $(75 \mu \mathrm{g} / \mathrm{mL})$ & 0.32 & 0.29 & 0.32 & 0.31 & 0.30 & 0.42 & 0.42 \\
\hline \multicolumn{8}{|l|}{ Inter-day precision* ( 3 days, $n=18$ ) } \\
\hline -low concentration $(25 \mu \mathrm{g} / \mathrm{mL})$ & 1.42 & 1.42 & 1.83 & 1.43 & 1.39 & 1.43 & 1.45 \\
\hline -high concentration $(75 \mu \mathrm{g} / \mathrm{mL})$ & 0.96 & 0.94 & 0.95 & 0.98 & 0.93 & 0.95 & 1.02 \\
\hline$\%$ Recovery $($ mean $\pm \mathrm{SD}, \mathrm{n}=3$ ) & $89.15 \pm 2.05$ & $94.89 \pm 4.72$ & $87.43 \pm 0.70$ & $91.13 \pm 1.69$ & $90.56 \pm 1.87$ & $84.71 \pm 2.66$ & $85.93 \pm 2.14$ \\
\hline Linear equation & $y=33,742+22,663$ & $y=38,094 x+24,417$ & $y=41,842 x+29,075$ & $y=42,971 x+30,789$ & $y=48,548 x+57,977$ & $y=25,400 x+15,550$ & $y=38,770 x+24,195$ \\
\hline Range $(\mu \mathrm{g} / \mathrm{mL})$ & $2-100$ & $2-100$ & $2-100$ & $2-100$ & $2-100$ & $2-100$ & $2-100$ \\
\hline Coefficient of correlation & 0.9997 & 0.9997 & 0.9997 & 0.9997 & 0.9995 & 0.9997 & 0.9997 \\
\hline $95 \% \mathrm{CI}$ of intercept & $-7,187.4-52,512.5$ & $-9,211.3-58,045.2$ & $-6,411.1-64,561.0$ & $-7,945.5-69,524.0$ & $2,836.3-113,117.2$ & $-4,602.3-35,701.7$ & $-7,138.6-55,529.6$ \\
\hline $\mathrm{LOD}(\mu \mathrm{g} / \mathrm{mL})$ & 1.35 & 1.35 & 1.30 & 1.38 & 1.73 & 1.21 & 1.23 \\
\hline LOQ $(\mu \mathrm{g} / \mathrm{mL})$ & 4.09 & 4.09 & 3.93 & 4.17 & 5.26 & 3.67 & 3.74 \\
\hline $\begin{array}{l}\text { Stability of standard solution } \\
(2 \text { days, mean } \pm S D, n=3)\end{array}$ & $100.15 \pm 1.44$ & $100.14 \pm 1.45$ & $99.93 \pm 1.45$ & $100.08 \pm 1.51$ & $100.10 \pm 1.43$ & $100.19 \pm 1.53$ & $100.42 \pm 1.53$ \\
\hline $\begin{array}{l}\text { Amount of compounds } \\
(\mathrm{mg} / \mathrm{g} \text { extract, } \text { mean } \pm \mathrm{SD}, \mathrm{n}=3)\end{array}$ & $27.60 \pm 0.25$ & $13.63 \pm 0.11$ & $15.82 \pm 0.13$ & $3.73 \pm 0.03$ & $21.72 \pm 0.19$ & $58.01 \pm 0.54$ & $36.76 \pm 0.20$ \\
\hline
\end{tabular}


Sungthong et.al., Rec. Nat. Prod. (2022) 16:4 358-369

Table 3. Docking score of the isolated compounds from C. harmandiana with TcAChE.

\begin{tabular}{|c|c|c|c|c|c|}
\hline Compounds & $\begin{array}{c}\mathrm{IC}_{50} \\
(\mu \mathrm{M} \pm \mathrm{SD})\end{array}$ & $\begin{array}{c}\text { Binding } \\
\text { energy } \\
(\mathrm{kcal} / \mathrm{mol})\end{array}$ & Pocket sites & Type of bonds & $\begin{array}{c}\text { Interacting residues } \\
\text { (Distance; } \AA \text { ) }\end{array}$ \\
\hline \multirow[t]{5}{*}{ Donepezil } & \multirow[t]{5}{*}{-} & \multirow[t]{5}{*}{-11.30} & PAS & $\pi-\pi$ interaction & Trp279 (4.3) \\
\hline & & & \multirow[t]{2}{*}{$\mathrm{ABP}$} & $\pi-\pi$ interaction & Phe290 (4.7) \\
\hline & & & & Hydrophobic & Phe290, Phe331 \\
\hline & & & \multirow[t]{2}{*}{ CAS } & $\pi-\pi$ interaction & Trp84 (3.9) \\
\hline & & & & Hydrophobic & Trp84, Phe330 \\
\hline \multirow{4}{*}{ Rivastigmine } & \multirow[t]{4}{*}{-} & \multirow{4}{*}{-7.04} & PAS & Hydrogen bond & Tyr121 (2.3) \\
\hline & & & $\mathrm{ABP}$ & Hydrophobic & Phe331 \\
\hline & & & CAS & $\pi-\pi$ interaction & Phe330 (3.8) \\
\hline & & & & Hydrophobic & Phe330 \\
\hline \multirow[t]{7}{*}{ Galantamine } & \multirow[t]{7}{*}{$1.06 \pm 0.07^{\mathrm{a}}$} & \multirow[t]{7}{*}{-9.84} & PAS & Hydrogen bond & Tyr121 (2.1) \\
\hline & & & $\mathrm{ABP}$ & $\pi-\pi$ interaction & Phe331 (4.8) \\
\hline & & & & Hydrophobic & Phe331 \\
\hline & & & CAS & Hydrogen bond & Phe330 (2.2) \\
\hline & & & & $\pi-\pi$ interaction & Phe330 (4.1) \\
\hline & & & & Hydrophobic & Phe330 \\
\hline & & & CT & Hydrogen bond & Ser200 (2.1) \\
\hline Tacrine & - & -7.34 & $\mathrm{ABP}$ & Hydrogen bond & Phe331 (2.2) \\
\hline \multirow[t]{4}{*}{1} & \multirow[t]{4}{*}{$106.94 \pm 1.22^{\mathrm{e}}$} & \multirow[t]{4}{*}{-8.47} & \multirow[t]{2}{*}{ PAS } & Hydrogen bond & Trp279 (1.7) \\
\hline & & & & Hydrophobic & Trp279 \\
\hline & & & \multirow{2}{*}{$\mathrm{ABP}$} & Hydrogen bond & $\operatorname{Arg} 289(2.0,2.3)$ \\
\hline & & & & Hydrophobic & Phe331 \\
\hline \multirow[t]{3}{*}{2} & \multirow{3}{*}{$104.06 \pm 0.75^{\mathrm{d}, \mathrm{e}}$} & \multirow[t]{3}{*}{-9.56} & \multirow[t]{2}{*}{ PAS } & Hydrogen bond & Asp72 (2.2) \\
\hline & & & & Hydrophobic & Ser122 \\
\hline & & & CAS & Hydrophobic & Trp84 \\
\hline \multirow[t]{2}{*}{3} & \multirow[t]{2}{*}{$99.44 \pm 2.57^{\mathrm{c}}$} & \multirow[t]{2}{*}{-9.20} & PAS & Hydrophobic & Ser122 \\
\hline & & & CAS & Hydrophobic & Trp84 \\
\hline 4 & $92.29 \pm 2.05^{\mathrm{b}}$ & -10.43 & PAS & Hydrogen bond & Tyr121 (3.3) \\
\hline & & & & $\pi-\pi$ interaction & Tyr334 (4.2) \\
\hline & & & & Hydrophobic & Tyr334 \\
\hline & & & CAS & Hydrogen bond & Phe330 (2.1) \\
\hline 5 & $99.88 \pm 2.50^{\mathrm{c}, \mathrm{d}}$ & -7.83 & PAS & Hydrogen bond & Trp279 (2.1) \\
\hline & & & & Hydrophobic & Tyr334 \\
\hline & & & $\mathrm{ABP}$ & Hydrogen bond & Ser286 (2.0) \\
\hline & & & & $\pi-\pi$ interaction & Phe331 (4.0) \\
\hline & & & & Hydrophobic & Phe331 \\
\hline & & & CAS & Hydrophobic & Phe330 \\
\hline 6 & $91.52 \pm 0.23^{\mathrm{b}}$ & -8.84 & PAS & Hydrogen bond & Trp279 (1.9) \\
\hline & & & & $\pi-\pi$ interaction & Tyr334 (4.0) \\
\hline & & & & Hydrophobic & Tyr70, Tyr334 \\
\hline & & & $\mathrm{ABP}$ & Hydrogen bond & Phe288 (1.8) \\
\hline & & & CAS & Hydrophobic & Phe330 \\
\hline 7 & $91.83 \pm 1.20^{\mathrm{b}}$ & -8.14 & PAS & Hydrogen bond & $\operatorname{Trp} 279(2.2)$ \\
\hline & & & & $\pi-\pi$ interaction & Tyr334 (3.7) \\
\hline & & & & Hydrophobic & Trp279, Tyr334 \\
\hline & & & $\mathrm{ABP}$ & Hydrogen bond & Phe288 (2.1) \\
\hline
\end{tabular}


AChE inhibitory effects of coumarins and carbazole alkaloids from C. harmandiana

In summary, the major binding site of all the compounds was the PAS site. There are previous reports that binding to the PAS of the AChE decreases the accumulating effects of the enzyme on the $\beta$ amyloid peptide, which are possibly involved in decreasing the accumulation of $\beta$-amyloid. Therefore, in vitro study for their activity as anti-Alzheimer's disease on targeted enzymes (i.e. $\beta$-secretase: BACE1) will feature in future studies. Moreover, coumarins core structure especially $\mathbf{4}$ has effects on docking score and interactions with the amino acid in the active site, although $\mathbf{4}$ expressed quite high cLogP but could not penetrate across the blood brain barrier and would therefore require its chemical structure to be modified for it to become to be a candidate molecule for further development as an antiAlzheimer's substance.

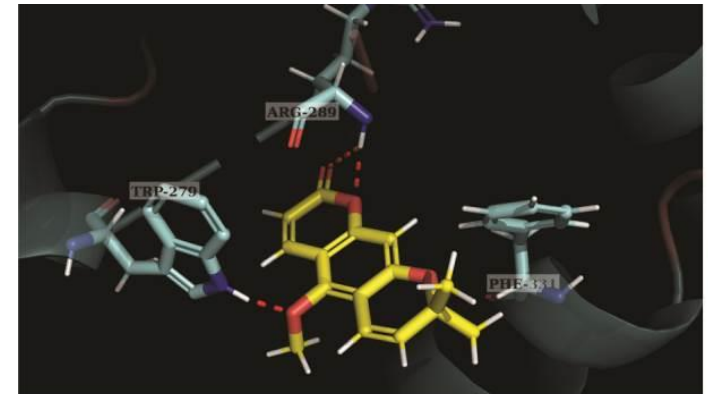

(a)

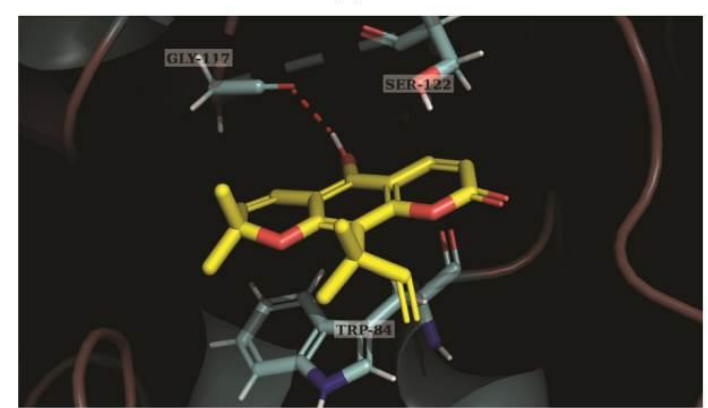

(c)

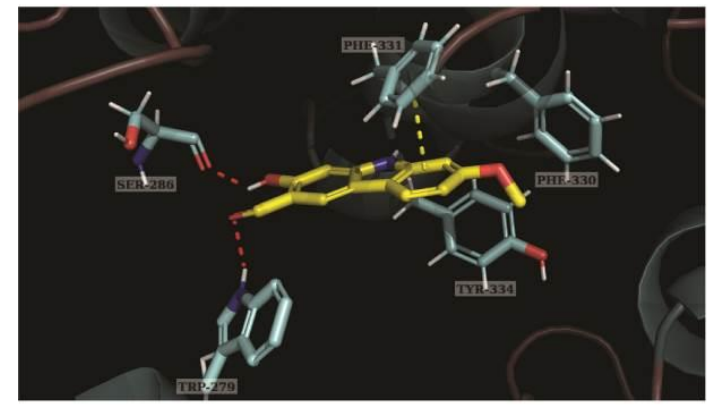

(e)

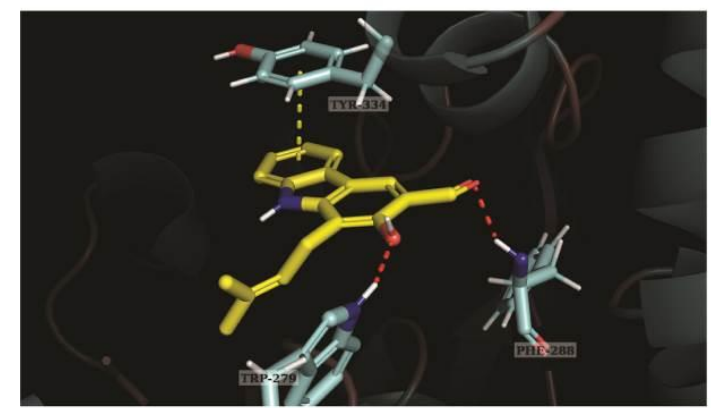

(g)

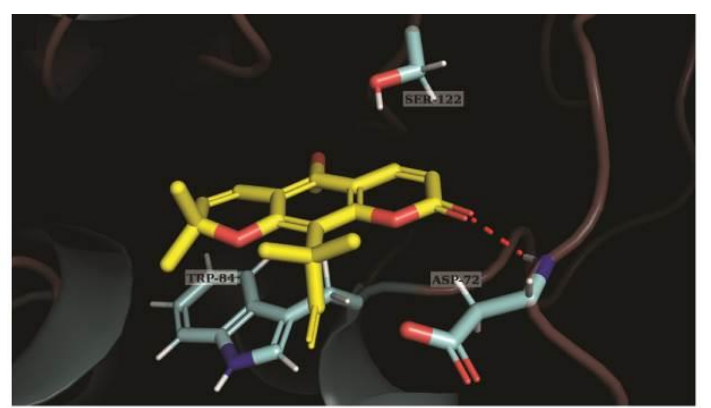

(b)

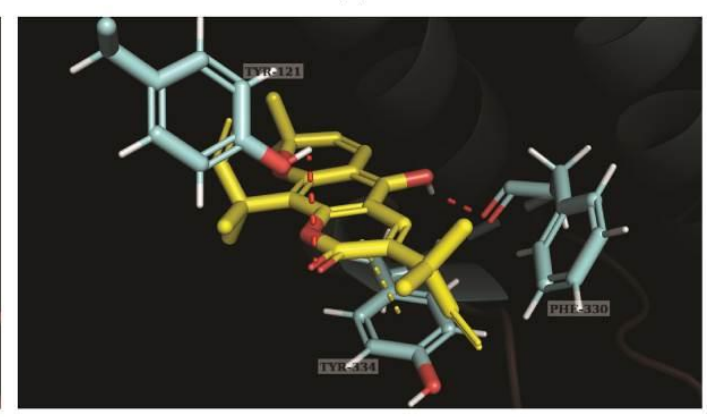

(d)

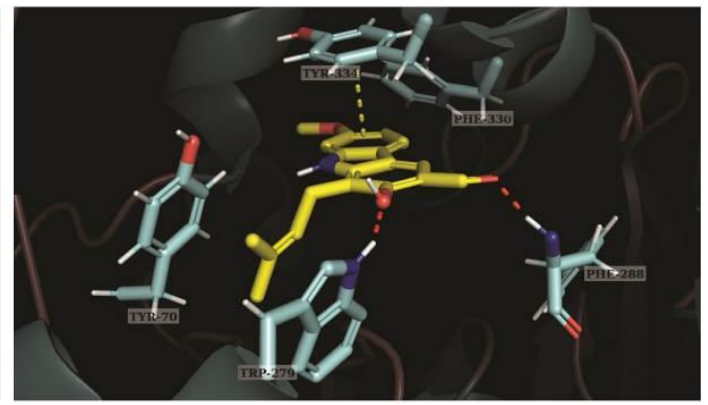

(f)

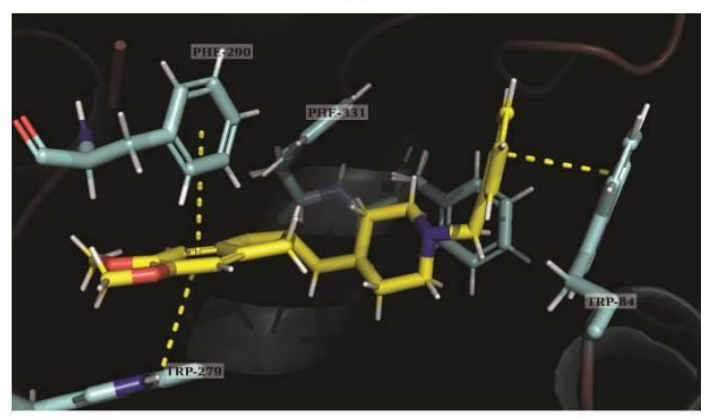

(h)

Figure 2. $T$. californica acetylcholinesterase ( $T c \mathrm{AChE}$ ) complexed with a) xanthoxyletin (1) b) dentatin (2) c) nordentatin (3) d) clausarin (4) e) 7-methoxymukonal (5) f) 7-methoxyheptaphylline (6) g) heptaphylline (7) and h) Donepezil 


\subsection{ADME Properties}

The drug likeness properties and pharmacokinetics were characterized by SwissADME program. Lipinski's rule is a rule to evaluate whether compound has physicochemical properties that would make it likely to be an orally active drug in humans. The positive control of AChE inhibitor agents (donepezil) and all isolated coumarins and carbazole alkaloids satisfy Lipinski's, as shown in Table 4. According to ADME predictions, these isolated compounds (1-7) displayed the flexible bond with NORTB value less than 10 (Table 4). Molecular polar surface area (TPSA) of all compounds met the criteria $(\leq 140)$, which indicated that all compounds had a good oral bioavailability, and the percentage oral absorption was in the range 87.5-92.20\%. Hence, all compounds (1-7) have good absorption and oral bioavailability. In addition, all compounds are not the substrates of P-gp which do not extrude in this efflux process and would not effect on concomitant administration of P-gp inhibitors or inducers [31]. Almost all compounds can easily cross the blood-brain barrier, except compound 4 (Table 5). Besides, all compounds can be mainly metabolized by cytochrome P450 enzymes (CYP).

Table 4. Screening of Lipinski's properties of the isolated compounds from C. harmandiana

\begin{tabular}{|c|c|c|c|c|c|c|c|c|}
\hline Compounds & $\mathbf{M W}^{\mathbf{a}}$ & $\operatorname{cLog} \mathrm{P}^{b}$ & cLogS $S^{c}$ & TPSA $^{\mathrm{d}}$ & NORTB $^{\text {e }}$ & $\mathbf{H B A}^{\mathrm{f}}$ & HBD $^{\mathrm{g}}$ & $\begin{array}{l}\text { Lipinski's } \\
\text { violation }\end{array}$ \\
\hline Criteria & $<500$ & - & - & $\leq 140$ & $\leq 10$ & $<5$ & $<10$ & $\leq 0$ \\
\hline Donepezil & 379.49 & 4.00 & -4.81 & 38.77 & 6 & 4 & 0 & 0 \\
\hline 1 & 258.27 & 2.80 & -3.53 & 48.67 & 1 & 4 & 0 & 0 \\
\hline 2 & 326.39 & 4.17 & -4.97 & 48.67 & 3 & 4 & 0 & 0 \\
\hline 3 & 312.36 & 3.78 & -4.75 & 59.67 & 2 & 4 & 1 & 0 \\
\hline 4 & 380.48 & 5.22 & -6.22 & 59.67 & 4 & 4 & 1 & 0 \\
\hline 5 & 241.24 & 2.36 & -3.64 & 62.32 & 2 & 3 & 2 & 0 \\
\hline 6 & 309.36 & 3.90 & -5.02 & 62.32 & 4 & 3 & 2 & 0 \\
\hline 7 & 279.33 & 3.88 & -4.96 & 53.09 & 3 & 2 & 2 & 0 \\
\hline
\end{tabular}

${ }^{a} \mathrm{MW}$ : molecular weight. ${ }^{\mathrm{b}} \mathrm{cLogP}$ : calculated octanol/water partition coefficient. ${ }^{\mathrm{c}} \mathrm{cLogS}$ : solubility parameter. ${ }^{\mathrm{d}}$ TPSA: topological polar surface area. ${ }^{\mathrm{e} N O R T B}$ : Number of freely rotatable bonds. ${ }^{\mathrm{f}} \mathrm{HBA}$ : Number of hydrogen bond acceptors. ' $\mathrm{HBD}$ : Number of hydrogen bond donors. 'hipinski's violation: 0 violation is good.

Table 5. ADME prediction of the isolated compounds from C. harmandiana

\begin{tabular}{ccccccccc}
\hline Compounds & \%ABS & $\begin{array}{c}\text { BBB } \\
\text { permeant }\end{array}$ & $\begin{array}{c}\text { P-gp } \\
\text { substrate }\end{array}$ & $\begin{array}{c}\text { CYP } \\
\text { 1A2 Inh }\end{array}$ & $\begin{array}{c}\text { CYP } \\
\text { 2C19 Inh }\end{array}$ & $\begin{array}{c}\text { CYP } \\
\text { 2C9 Inh }\end{array}$ & $\begin{array}{c}\text { CYP } \\
\text { 2D6 Inh }\end{array}$ & $\begin{array}{c}\text { CYP } \\
\text { 3A4 Inh }\end{array}$ \\
\hline Donepezil & 95.62 & Yes & No & Yes & Yes & Yes & No & No \\
$\mathbf{1}$ & 92.21 & Yes & No & Yes & Yes & Yes & No & No \\
$\mathbf{2}$ & 92.21 & Yes & No & Yes & Yes & Yes & No & No \\
$\mathbf{3}$ & 88.41 & Yes & No & Yes & Yes & Yes & No & No \\
$\mathbf{4}$ & 88.41 & No & No & No & No & Yes & No & Yes \\
$\mathbf{5}$ & 87.50 & Yes & No & Yes & No & No & Yes & Yes \\
$\mathbf{6}$ & 87.50 & Yes & No & Yes & Yes & Yes & Yes & No \\
$\mathbf{7}$ & 90.68 & Yes & No & Yes & Yes & Yes & Yes & No \\
\hline$\%$ ABS $=109-[0.345 \times$ TPSA] $[32]$ & & & & & &
\end{tabular}

\section{Conclusions}

The molecular docking result showed that $\mathbf{4}$ formed two strong H-bonds between $\mathrm{O}$-atoms of pyranone ring and Tyr121 at PAS and another one is between hydroxy group at R3 and Phe330 at CAS. Interestingly, carbazole alkaloids, $\mathbf{6}$ and $\mathbf{7}$ displayed the similar binding energy and $\mathrm{IC}_{50}$ values. Their binding interactions also established the same pocket sites at PAS and ABP. In summary, we could fine 
AChE inhibitory effects of coumarins and carbazole alkaloids from C. harmandiana

that, O-atom at R3 of coumarins is a key role in H-bonds interactions with PAS of TcAChE enzyme. 1,1-dimethyl-2-propenyl group at R1 and R2 can stabilize binding affinity between the core structure of coumarins and TcAChE enzyme at the PAS pocket site. Meanwhile, aromatic rings of carbazole alkaloids were found favourably interact via $\pi-\pi$ interaction with PAS or ABP pocket sites. The O-atom from both hydroxyl and formyl groups were also present at the main binding pocket with $T c \mathrm{AChE}$ enzyme. Besides the major binding site of 1-7 was the PAS pocket site.

\section{Acknowledgments}

This research was funded by Thailand Research Fund, Grant No. MRG5180175 and DBG6080006, Thailand. The authors would like to thank Prof. Dr. Adrian Roderick Plant, Faculty of Sciences, Mahasarakham University for English editorial assistance and Assist. Prof. Dr. Chamnan Pattarapanich to give advice for this project.

\section{ORCID}

Bunleu Sungthong: 0000-0001-8697-0718

Kingkaeo Sithon: 0000-0002-2637-8437

Panyada Panyatip: 0000-0003-4675-7132

Sarin Tadtong: 0000-0003-2229-1999

Nadtanet Nunthaboot: 0000-0003-3939-3879

Ploenthip Puthongking: 0000-0002-5069-2071

\section{References}

[1] A. Sanabria-Castro, I. Alvarado-Echeverría and C. Monge-Bonilla (2017). Molecular pathogenesis of Alzheimer's disease: an update.,Ann. Neurosci. 24, 46-54.

[2] R. T. Bartus (1978). Evidence for a direct cholinergic involvement in the scopolamine-induced amnesia in monkeys: Effects of concurrent administration of physostigmine and methylphenidate with scopolamine, Pharmacol. Biochem. Behav. 9, 833-836.

[3] G. D. Stanciu, A. Luca, R. N. Rusu, V. Bild, S. I. B. Chiriac, C. Solcan, W. Bild and D. C. Ababei (2020). Alzheimer's disease pharmacotherapy in relation to cholinergic system involvement, Biomolecules 10,121.

[4] S. K. Yusufzai, M. S. Khan, O. Sulaiman, H. Osman and D. N. Lamjin (2018). Molecular docking studies of coumarin hybrids as potential acetylcholinesterase, butyrylcholinesterase, monoamine oxidase A/B and $\beta$-amyloid inhibitors for Alzheimer's disease, Chem. Cent J. 12, 1-57.

[5] F. Sonmez, B. Z. Kurt, I. Gazioglu, L. Basile, A. Dag, V. Cappello, T. Ginex, M. Kucukislamoglu and S. Guccione (2017). Design, synthesis and docking study of novel coumarin ligands as potential selective acetylcholinesterase inhibitors, J. Enzyme Inhib. Med. Chem. 32, 285-297.

[6] N. C. Inestrosa, A. Alvarez, C. A. Pérez, R. D. Moreno, M. Vicente, C. Linker, O. I. Casanueva, C. Soto and J. Garrido (1996). Acetylcholinesterase accelerates assembly of amyloid- $\beta$-peptides into Alzheimer's fibrils: Possible role of the peripheral site of the enzyme, Neuron 16, 881-891.

[7] Y. P. Liu, J. M. Guo, Y. Y. Liu, S. Hu, G. Yan, L. Qiang and Y.H. Fu (2019). Carbazole alkaloids with potential neuroprotective activities from the fruits of Clausena lansium. J. Agric. Food. Chem. 67, 57645771.

[8] H. Liu, C. J. Li, J. Z. Yang, N. Ning, Y. K. Si, L. Li, N. H. Chen, Q. Zhao and D. M. Zhang (2012). Carbazole alkaloids from the stems of Clausena lansium. J. Nat. Prod. 75, 677-682.

[9] J. Liu, Y. Q. Du, C. J. Li, L. Li, F. Y. Chen, J. Z. Yang, N. H. Chen and D. M. Zhang (2017). Alkaloids from the stems of Clausena lansium and their neuroprotective activity, RSC Advances. 7, 35417-35425.

[10] J. Wangboonskul, A. Tunsirikongkon and W. Sasithornwetchakun (2015). Simultaneous determination of nine analytes in Clausena harmandiana Pierre. by new developed high-performance liquid chromatography method and the influence of locations in Thailand on level of nordentatin and dentatin, Pharmacogn. Mag. 11, 1-7.

[11] P. Jantakoon, S. Tadtong and P. Puthongking (2012). Neuritogenic and antioxidant activities of nordentatin from Clausena harmandiana, JAASP. 1, 180-186.

[12] S. Y. Kang, K. Y. Lee, S. H. Sung, M. J. Park and Y. C. Kim (2001). Coumarins isolated from Angelica gigas inhibit acetylcholinesterase: Structure-activity relationships, J. Nat .Prod. 64, 683-685. 
[13] F. U. Rehman, M. F. Khan, I. Khan and Roohullah (2013). Molecular interactions of an alkaloid euchrestifoline as a new acetylcholinesterase inhibitor, Bangladesh J. Pharmacol.8, 361-364.

[14] N. S. Kumar, P. K. Mukherjee, S. Bhadra, B. P. Saha and B. C. Pal (2010). Acetylcholinesterase inhibitory potential of a carbazole alkaloid, mahanimbine, from Murraya koenigii. Phyther. Res. 24, 629631.

[15] P. Rodanant, R. Surarit, S. Laphookhieo and J. Kuvatanasuchati (2015). In vitro evaluation of the antibacterial and anti-inflammation activities of Clausena lansium (Lour.) skeels, Songklanakarin J. Sci. Technol. 37, 43-48.

[16] S. Rosselli, A. Maggio, G. Bellone, C. Formisano, A. Basile, C. Cicala, A. Alfieri, N. Mascolo and M. Bruno (2007). Antibacterial and anticoagulant activities of coumarins isolated from the flowers of Magydaris tomentosa, Planta Med. 73, 116-120.

[17] T. M. Ngoc, N. X. Nhiem, N. M. Khoi, D. C. Son, T. V. Hung and P. V. Kiem (2014). A new coumarin and cytotoxic activities of constituents from Cinnamomum cassia, Nat. Prod. Commun. 9, 487-488.

[18] P. Jantamat, N. Weerapreeyakul and P. Puthongking (2019). Cytotoxicity and apoptosis induction of coumarins and carbazole alkaloids from Clausena harmandiana, Molecules.24, 1-14.

[19] T. Ojala, S. Remes, P. Haansuu, H. Vuorela, R. Hiltunen, K. Haahtela and P.Vuorela (2000). Antimicrobial activity of some coumarin containing herbal plants growing in Finland, $J$. Ethnopharmacol. 73, 299-305.

[20] International Council for Harmonisation (2005). Switzerland: ICH guidelines, validation of analytical procedures: text and methodology Q2(R1). [cited 2020 Jun 29] Available from: http://www.ich.org.

[21] T. Petrachaianan, S. Chaiyasirisuwan, S. Athikomkulchai and V. Sareedenchai (2019). Screening of acetylcholinesterase inhibitory activity in essential oil from Myrtaceae, TJPS. 43, 63-68.

[22] G. Kryger, I. Silman and J. L. Sussman (1999). Structure of acetylcholinesterase complexed with E2020 (Aricept $\rho$ ): Implications for the design of new anti-Alzheimer drugs, Structure.7, 297-307.

[23] M. F. Sanner (1999). Python: A programming language for software integration and development, J. Mol. Graph. Model. 17, 57-61.

[24] G. M. Morris, R. Huey, W. Lindstrom, M. F. Sanner, R. K. Belew, D. S. Goodsell and A.J. Olson (2010). AutoDock4 and AutoDockTools4: Automated docking with selective receptor flexibility, J. Comput. Chem. 30, 2785-2791.

[25] H. R. Mashhadi, H. M. Shanechi, C. Lucas (2003). A new genetic algorithm with Lamarckian individual learning for generation scheduling, IEEE Trans. Power. Syst. 18, 1181-1186.

[26] N. M. O’Boyle, M. Banck, C. A. James, C. Morley, T. Vandermeersch and G. R. Hutchison (2011). Open Babel: An open chemical toolbox, Cheminform 3, 1-14.

[27] A. Daina, O. Michielin and V. Zoete (2017). SwissADME: A free web tool to evaluate pharmacokinetics, drug-likeness and medicinal chemistry friendliness of small molecules, Sci Rep. 3, 1-13

[28] I. Cruz, P. Puthongking, S. Cravo, A. Palmeira, H. Cidade, M. Pinto and E. Sousa (2017). Xanthone and flavone derivatives as dual agents with acetylcholinesterase inhibition and antioxidant activity as potential anti-Alzheimer agents, J. Chem. 2017, 1-16.

[29] M. Y. Ali, S. H. Seong, H. A. Jung, S. Jannat and J. S. Choi (2018). Kinetics and molecular docking of dihydroxanthyletin-type coumarins from Angelica decursiva that inhibit cholinesterase and BACE1, Arch. Pharm. Res. 41, 753-764.

[30] B. K. Shin, H. M. Kim, S. Lee and J. Han (2008). Axial conformation of 3-methyl-2-butenoyl group in pyranocoumarin ring endows biological activity of (+)-Decursin, Bull. Korean. Chem. Soc. 29, 15721574.

[31] S. Mollazadeh, A. Sahebkar, F. Hadizadeh, J. Behravan and S. Arabzadeh (2018). Structural and functional aspects of P-glycoprotein and its inhibitors, Life Sci. 214, 118-123.

[32] Y. H. Zhao, M. H. Abraham, J. Le, A. Hersey, C. N. Luscombe, G. Beck, B. Sherborne and I. Cooper (2002). Rate-limited steps of human oral absorption and QSAR studies, Pharm. Res. 19, 1446-1457.

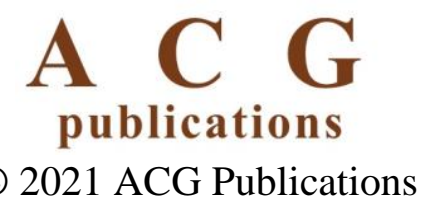

